\title{
Literatur
}

1. Graef, V., P. Jobst und HJ. Staudinger, diese Z. 6,159 (1968). - 2. Hudson, B., J. Coghlan, A. Dulmanis, M. Wintour und I. Ekker, Austral. J. Exper. Biol. 41, 235 (1963). - 3. RionDEL, A., J. F. TAIt, M. Gut, S. A. S. Tait, E. Joachim und B. LitrLe, J. Clin. Endocr. Metab., Springfield 23, 620 (1963). 4. Burger, H. G., J. R. Kent und A. E. Kelite, J. Clin. Endocr. Metab., Springfield 24, 432 (1964). - 5. BrownIE, A. C., H. J. van der Molen, E. E. Nishizawa und K. B. Erk-Nes, J. Clin. Endocr. Metab., Springfield 24, 1091 (1964). - 6. NAKAGAwA, K.,
N. McNiven, E. Forchielli, A. Vermeulen und R. I. DorfMAN, Steroids 7, 329 (1966). - 7. Finkelstern, M., E. Forchielli und R. I. Dorfman, J. Clin. Endocr. Metab., Springfield 21, 98 (1961). - 8. Forchielli, E., G. Sorcini, M. S. Nightingale, N. Brust, R. I. Dorfman, W. H. Perloff und G. Jacobson, Analyt. Biochem. (New York) 5, 416 (1963). - 9. Horton, R., T. Kato und R. Sherins, Stetoids 10, 245 (1967). - 10. Oertel, G. W., Acta endocr. K'hvn 37, 237 (1961).

Prof. Dr. Hj. Staudinger $63 \mathrm{Gießen}$

Friedrichstr. 24

\section{Lactat- und Pyruvatkonzentrationen im Blut während der Acidose des Neugeborenen}

Von D. KaISER und E. WERner

Aus der Kinderklinik des Städt. Rudolf-Virchow-Krankenbauses Berlin (Ärztl. Direktor: Prof. Dr. E. Werner)

(Eingegangen am 2. April 1968)

Herrn Prof. Dr. Dr. Ernst Schütte qum 60. Geburtstag geividmet

An 18 rcifen Neugeborenen wurde festgestellt, daß die 48 Stdn. dauernde metabolische Acidose korreliert ist mit den Blutlactatwerten. Außerdem fand sich - trotz ausreichenden $\mathrm{O}_{2}$-Angebotes - ein erhöhter Reduktionszustand der Gewebe in diesem Lebensabschnitt. Es konnte gezeigt werden, daß die Bestimmung von Lactat und Pyruvat auch eine klinisch-diagnostische Aussagekraft für die postnatale Periods: besitzt.

A study of 18 mature, newly born infants showed that the metabolic acidosis, which lasts for 48 hrs., is correlated with the concentration of lactate in the blood. Furthermore, there was an increase in the reduction state of the tissue during this period despite a sufficient supply of oxygen. It was shown that the determination of lactate and pyruvate can also be used for diagnostic purposes during the postnatal period.

Störungen des Säure-Basen-Gleichgewichts in der perinatalen Periode sind für den Geburtshelfer und den Pädiater von großem Interesse, weil nach neueren Anschauungen (1) die Acidose weitgehend bestimmt: 1. Den Zeitpunkt aktiven geburtshilflichen Eingreifens 2. Art und Ausmaß der post partum einzuleitenden Maßnahmen der Acidosebekämpfung und damit 3. die Überlebenschancen des Neugeborenen.

Beim reifen Neugeborenen ist die respiratorische Komponente der Acidose schon 60 bis 120 Min. nach der Abnabelung ausgeglichen, wie aus den arteriellen $\mathrm{P}_{\mathrm{CO}_{2}}$-Werten (30-40 Torr) $\mathrm{zu}$ ersehen ist (2).

Unabhängig hiervon verläuft die metabolische Acidose. Viele Untersuchungen der letzten Jahre zeigten, daß sie mit einem Anstieg der Blutlactatkonzentrationen einhergeht. Insbesondere der Zusammenhang zwischen $\operatorname{der} \mathrm{O}_{2}$-Versorgung einerseits, sowie den Lactatkonzen-' trationen und der metabolischen Acidose andererseits, schien uns von Interesse für die Postnatalperiode. Durch gleichzeitige Bestimmung des Säure-Basen-Status ( $\mathrm{pH}$, Standardbicarbonat, $\mathrm{P}_{\mathrm{co}_{2}}$ ), des $\mathrm{P}_{\mathrm{o}_{2}}$, sowie der Lactat- $\left(C_{I}\right)$ und Pyruvatkonzentrationen $\left(C_{P}\right)$ sollte festgestellt werden, wie beim reifen Neugeborenen die metabolische Acidose verläuft, welche Beziehungen zum Lactat bestehen und wie der Reduktionszustand der Gewebe - gemessen am Lactat-Pyruvatquotienten (L/P) $(3,4,5)$ - vom Blutsauerstoff-Angebot abhängt.
Wir fanden, daß die neonatale Acidose vorwiegend in einer Lactatanbäufung bestebt. Sie bält bis 48 Stdn. nach der Geburt an. Trotz ausreichenden $\mathrm{O}_{2}$-Angebotes ist gleichzeitig auch der Redultionszustand der Gevebe (L/P-Quotient) erböbt. Wir balten es für wabrscheinlich, daß dies zurückzufübren ist auf eine geringere Oxydationskapazität des Neugeborenen für Milchsäure.

Nach unserer Meinung stellt die Bestimmung von Lactat und Pyruvat eine wertvolle Bereicherung der Diagnostik in der Postnatalperiode dar.

\section{Methodik}

Die Untersuchungen wurden an 18 reifen, komplikationslos geborenen Säuglingen mit einem Durchschnittsgewicht von $3200 \mathrm{~g}$ über die ersten 48 Lebensstunden durchgeführt. Zur Standardisierung der Stoffwechselbedingungen wurden die Säuglinge eine Stunde vor den Messungen in einen $32^{\circ}$ warmen, d. h. indifferent temperierten Inkubator gelegt $(6,7)$. Aus der hyperaemisierten (Rubriment) und silikonisierten (Hemolube-Salbe) Ferse wurde Kapillarblut in heparinisierte Glasröhrchen entnommen. Zwei Röhrchen wurden zu Bestimmungen von $\mathrm{pH}$, Standardbicarbonat und $\mathrm{P}_{\mathrm{CO}_{2}}$ sowie des $\mathrm{P}_{\mathrm{O}_{2}}$ am Mikro-Kombi-Analysator (Fa. Eschweiler) verwendet.

Zur Lactat- und Pyruvatmessung wurden die ursprünglichen Methoden $(8,9)$ auf Halbmiktoverfahren adaptiert (EPPENDORF Mikrolitersystem) und wie folgt modifiziert: Aus dem Einstich perlendes Fersenblut wird lückenlos in zwei heparinisierte „EscHwEILER-Kapillaren" (etwa $140 \mu l$ ) aufgesaugt, sofort vollständig in eisgekühlte $0,6 \mathrm{~N} \mathrm{HClO}_{4}$ ausgeblasen, gut durchmischt und 60 Sek. bei $18000 \mathrm{~g}$ zentrifugiert. $200 \mu /$ des Überstandes werden mit 100 bis 
$120 \mu l 0,6 \mathrm{~N} \mathrm{KOH}$ auf $\mathrm{pH} 7,5$ bis 8,0 eingestellt (tüpfeln auf Merck-Indikatorpapier). Die zur Blutentnahme benutzte Kapillare wird dann leer und wassergefüllt gewogen und damit das Volumen ermittelt.

\section{Lactatbestimmung}

\section{Erforderliche Reagenzien}

1. Glycinpuffer: 7,5 g Glycin (Merck 4200), 5,2 g Hydrazinsulfat (Merck 4603) und 0,2 $\mathrm{g}$ EDTA (Merck 8418) in wenig dest. Wasser lösen, $51 \mathrm{~m} / 2 \mathrm{~N} \mathrm{NaOH}$ zugeben und mit dest. Wasser ad 100,0 auffüllen. pH auf 9,5 einstellen. Den Puffer in kleinen Einzelportionen einfrieren, da in flüssigem Zustand sehr instabil. 2. NAD (BoeHringer 15300$) 40 \mathrm{mg} / \mathrm{ml}$.

3. $\mathrm{LDH}^{1}$ ) (BOEHRINGER ELAC 15 371) Kristallsuspension $5 \mathrm{mg} / \mathrm{ml}$, mit bidest Wasser $1: 1$ verdünnen.

\section{Bestimmungsansatz}

Küvettenlänge $20 \mathrm{~mm}$. Meßstrahlung $334 \mathrm{~nm}$.

Der Reihe nach einpipettieren: (Doppelbestimmung)

$$
\begin{aligned}
& 200 \mu l \text { Glycinpuffer (s. o.) } \\
& 100 \mu l \text { bidest. Wasser } \\
& 10 \mu l \text { enteiweißten neutralisierten Extrakt } \\
& 5 \mu l \text { NAD } \\
& 100 \mu l \text { bidest. Wasser } \\
& \hline 415 \mu l \text { Endvolumen. }
\end{aligned}
$$

Die Vergleichsküvette enthält den gleichen Ansatz, statt des Extraktes werden $10 \mu l$ bidest. Wasser einpipettiert. Mit Polyäthylenstab gut durchmischen und nach Konstanz der Extinktion Ausgangswerte notieren, Reaktion starten mit $10 \mu l \mathrm{LDH}$, mișchen und nach 10-15 Min. Extinktion ablesen.

\section{Pyruvatbestimmung}

\section{Erforderliche Reagenzien:}

1. Triäthanolamin-Puffer (Boehringer 15325 TRA) $18,57 \mathrm{~g}$ Triäthanolamin- $\mathrm{HCl}$ in $1000 \mathrm{~m} /$ bidest. Wasser lösen und mit $2 \mathrm{~N}$ $\mathrm{NaOH}$ auf $\mathrm{pH} 7,6$ einstellen.

2. NADH (Boehringer DPNH 15142). Mit TriäthanolaminPuffer auf Konzentration 0,5 bis $1,0 \mathrm{mg} / \mathrm{m} l$ einstellen. Lösung täglich frisch ansetzen.

\section{Bestimmungsansatz}

Küvettenlänge $20 \mathrm{~mm}$. Meßstrahlung $334 \mathrm{~mm}$.

Der Reihe nach einpipettieren: (Doppelbestimmung)

$200 \mu l$ Triäthanolamin-Puffer

$100 \mu l$ enteiweißten neutralisierten Extrakt

$5 \mu l \mathrm{NADH}$

$$
\begin{aligned}
& 100 \mu l \text { Triäthanolamin-Puffer } \\
& \hline 405 \mu l \text { Endvolumen. }
\end{aligned}
$$

Gleichen Ansatz in der Vergleichsküvette, statt des Extraktes werden jedoch $100 \mu l$ bidest. Wasser einpipettiert. Mischen, Konstanz der Extinktion abwarten, Ausgangswerte notieren und Reaktion starten mit $5 \mu l$ LDH. Mischen. Nach 5-10 Min. Extinktion ablesen.

\section{Berechnung der Lactat- und Pyruvat-Konzentration}

$$
\begin{aligned}
\mathrm{c}_{\mathrm{E}} & =\frac{\mathrm{E} \cdot \mathrm{V}_{1}}{\varepsilon \cdot \mathrm{V}_{2}} \text { Konzentration in } \mu \mathrm{Mol} / \mathrm{m} l \text { Extrakt } \\
\mathrm{c}_{\mathrm{B}} & =\mathrm{c}_{\mathrm{E}} \cdot \mathrm{F} \text { Konzentration in } \mu \mathrm{Mol} / \mathrm{m} / \mathrm{Blut} \\
\mathrm{F} & =\frac{\left(\mathrm{HClO}_{4}+\mathrm{Blut}\right) \cdot(\text { Extrakt }+\mathrm{KOH})}{(\text { Blut }) \cdot(\text { Extrakt })}
\end{aligned}
$$

1) Abkürqung: $\mathrm{LDH}=$ Lactatdehydrogenase (E.C. 1.1.1.27).
$\mathrm{V}_{1}=$ Endvolumen der Küvette

$\mathrm{V}_{2}=$ eingesetztes Extraktvolumen

$\varepsilon=6,09 \cdot 10^{6} \mathrm{~cm}^{2} / \mathrm{Mol}$ bei $334 \mathrm{~nm}$

$\mathrm{E}=$ Extinktion (Vollwert - Blindwert)

$\mathrm{HClO}_{4}=$ vorgelegtes Perchlorsäurevolumen

Blut $=$ einpipettiertes Blutvolumen

Extrakt $=$ Volumen des zu neutralisierenden Extraktes

$\mathrm{KOH}=$ zur Neutralisation erforderliches Volumen 0,6N Kalilauge.

\section{Bestimmung des fötalen Hämoglobins $\mathrm{HbF}$}

Fötales Hämoglobin wurde als alkaliresistentes $\mathrm{Hb}$ nach der Methode von BETkE (10) photometrisch bestimmt.

\section{Ergebnisse}

Die Mittelwerte des $\mathrm{pH}$ und Standardbicarbonats zeigen, $\mathrm{da} \beta$ eine anfangs bestehende metabolische Acidose sich bis zum Ende des zweiten Lebenstages normalisiert hat. Im gleichen Zeitraum fällt Lactat im Blut von $2,5 \mu \mathrm{Mol} / \mathrm{m} l$ auf $1,4 \mu \mathrm{Mol} / \mathrm{ml}$, während Pyruvat mit Konzentrationen um $0,09 \mu \mathrm{Mol} / \mathrm{ml}$ ziemlich konstant bleibt. Steigendes Standardbicarbonat, d. h. abnehmende metabolische Acidose, ist korreliert mit fallenden Lactatwerten. Die Regressionsgerade hat die Gleichung:

$$
\begin{gathered}
\mathrm{St}\left[\mathrm{HCO}_{3}\right]^{-}=-16,15\left(\mathrm{C}_{\mathrm{L}}+\mathrm{C}_{\mathrm{P}}\right) \\
\mathrm{r}=0,935 \quad \mathrm{p}<0,001
\end{gathered}
$$

Vergleiche hierzu Abbildung 1.
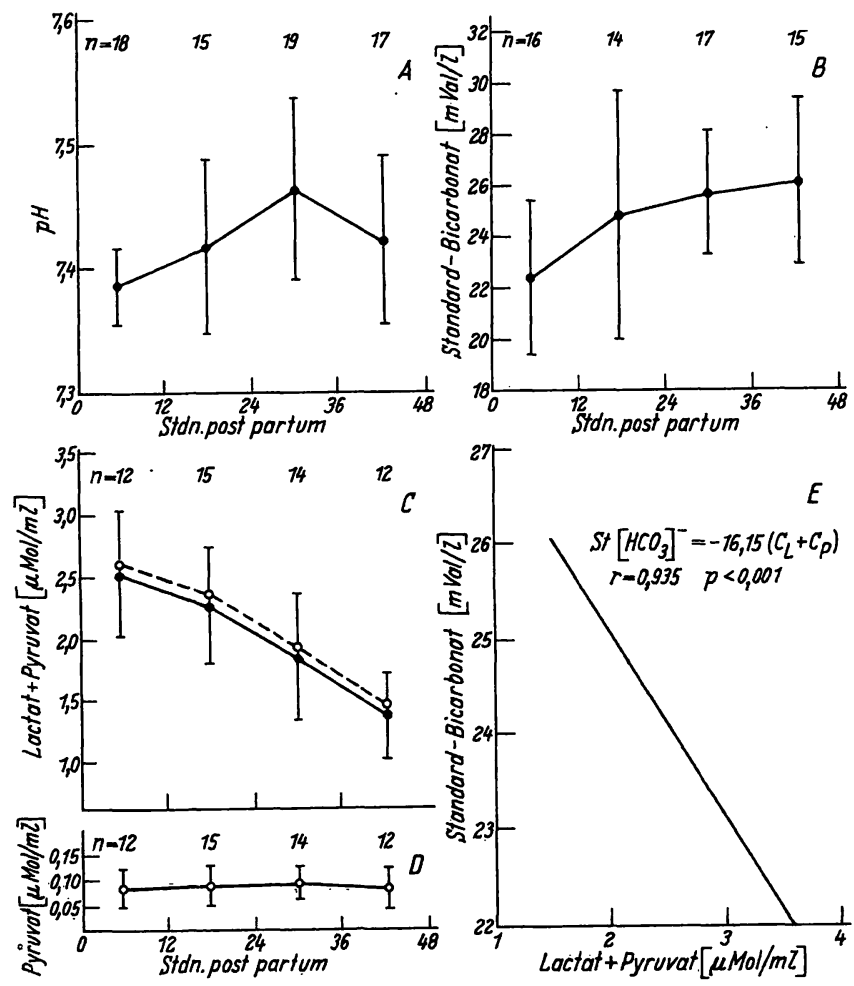

Abb. 1

A: Verlauf des aktuellen $\mathrm{pH}$

B: Verlauf des Standardbicarbonates

C: Verlauf der Lactatkonzentrationen $(\bullet-\circ)\left(C_{L}\right)$, und der Summe der Lactat- und Pyruvatkonzentrationen $\left(o_{-}-0\right)\left(C_{L}+C_{P}\right)$

D: Verlauf der Pyruvatkonzentrationen $(\circ)\left(C_{P}\right)$

E: Regressionsgerade far die negative Korrelation ( $p<0,001)$ zwischen dem Standardbicarbonat und der Summe der Lactatund Pyruvatkonzentrationen $\left(C_{L}+C_{P}\right)$. 
Auch der Quotient Lactat/Pyruvat zeigt über die Zeit fallende Tendenz (Tab. 1).

Tab. 1

Lactat/Pyruvat-Quotient zu verschiedenen Zeiten nach der Geburt

\begin{tabular}{ccccc}
\hline $\begin{array}{c}\text { Stdn. post } \\
\text { partum }\end{array}$ & $0-12$ & $12-24$ & $24-36$ & $36-48$ \\
\hline L/P & 28 & 23 & 21 & 16 \\
\hline
\end{tabular}

In Ä̈bildung 2 sind die L/P-Quotienten gegen den Blut- $\mathrm{P}_{\mathrm{O}_{2}}$ aufgetragen. Die Anordnung der Meßpunkte ist regellos und läßt keine Abhängigkeit des $\mathrm{L} / \mathrm{P}-$ Quotienten vom $\mathrm{P}_{\mathrm{O}_{2}}$ erkennen. Jedoch fällt auf, daß die L/P-Werte ausnahmslos über dem für ruhende oder ausreichend mit Sauerstoff versorgte Gewebe ermittelten Quotienten von 10 liegen $(3,4)$.

Die Tabelle 2 zeigt den Verlauf von $C_{I}, C_{P}, L / P$, Standardbicarbonat, $\mathrm{P}_{\mathrm{O}_{2}}$ und Hauttemperatur des Oberschenkels $\left(T_{H}\right)$ bei drei Neugeborenen.

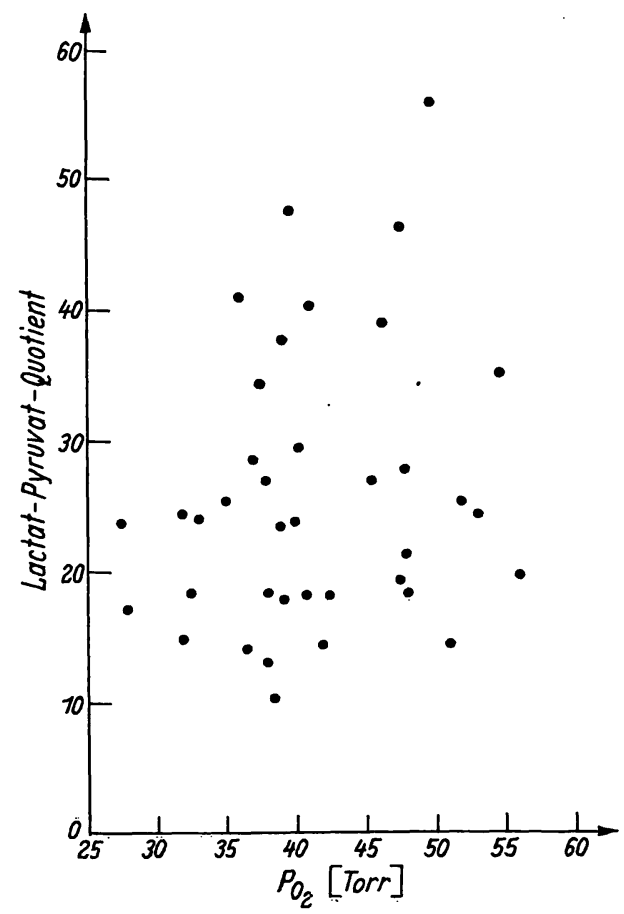

Abb. 2

Die L/P-Quotienten liegen in den ersten 2 Lebenstagen über 10 (Wert für ruhende, reife, ausreichend $\mathrm{O}_{2}$-versorgte Gewebe). Eine Abhängigkeit vom $\mathrm{P}_{\mathrm{O}_{2}}$ des Kapiḷ larblutes besteht nicht.

\section{Diskussion}

Reife, ruhende, ausreichend mit Sauerstoff versorgte Gewebe weisen einen L/P-Quotienten von 10 auf $(3,4)$. Unter $\mathrm{O}_{2}$-Mangel steigt der L/P-Quotient durch vermehrte Lactatbildung (Exzeßlactat) (4, 5). Dieser erhöhte Reduktionszustand im Gewebe ist im allgemeinen erst nachweisbar bei Absinken des atteriellen $\mathrm{P}_{\mathrm{O}_{2}}$ unter 30 Torr (5). Im Gegensatz hierzu liegen die $\mathrm{P}_{\mathrm{O}_{2}}$ des Fersenkapillarblutes unserer Neugeborenen im Mittel bei 42 Torr (max. Streuung 27,5-55,5 Torr) und unterschreiten nur in zwei Fällen 30 Torr (Abb. 2). Die mittlere Sauerstoffkonzentration des Blutes unter diesen $\mathrm{O}_{2}$-Drucken berechnet sich zu $19,5 \mathrm{ml} \mathrm{O}_{2} /$ $100 \mathrm{~m} l$ Blut (max. Streuung $16,2-22,0 \mathrm{~m} l \mathrm{O}_{2} / 100 \mathrm{~m} l$ Blut). Der Berechnung liegt die Sauerstoff-Dissoziationskurve für das Blut unserer Neugeborenen zugrunde: Bei einem Gesamt-Hb von $18 \mathrm{~g} / 100 \mathrm{~m} l$ besteht es zu $68 \%(12,24 \mathrm{~g} / 100 \mathrm{~m} l)$ aus $\mathrm{HbF}$ und $\mathrm{zu} 32 \%(5,76 \mathrm{~g} /$ $100 \mathrm{ml}$ ) aus $\mathrm{HbA}$ (Erwachsenen-Hämoglobin).

$\mathrm{Da}$ Erwachsenenblut $(14 \mathrm{~g} / 100 \mathrm{~m} /)$ unter gleichen Bedingungen nur $13,5 \mathrm{~m} l \mathrm{O}_{2} / 100 \mathrm{~m} l$ Blut (max. Schwankung $11-15 \mathrm{~m} l \mathrm{O}_{2} / 100 \mathrm{~m} l$ Blut) enthält, ist die Sauerstoffkonzentration im Neugeborenenblut wesentlich höher als beim Erwachsenen und gewährleistet im interessierenden $\mathrm{P}_{\mathrm{O}_{2}}$-Bereich ein voll ausreichendes $\mathrm{O}_{2}$-Angebot.

Dennoch lassen die hohen Lactatwerte und L/PQuotienten auf einen erhöhten Reduktionszustand bis 48 Stdn. nach der Geburt schließen.

Diese Befunde lassen sich am ehesten deuten durch eine gegenüber dem älteren Säugling und dem Erwachsenen verminderte Oxydationskapazität für Milchsäure in der Postnatalperiode. Hierfür sprechen folgende Tatsachen: Die Höhe des initialen Blutlactatspiegels des Neugeborenen hängt weitgehend von der Wehentätigkeit des Myometriums ab, so daß in erster Linie an eine diaplazentare materno-fötale Übertragung von Exzeßlactat gedacht werden muß $(11,12)$; jedoch fällt auf, $\mathrm{da}$ die Lactatelimination nach unseren Ergebnissen $48 \mathrm{Stdn}$. benötigen würde, wogegen Exzeßlactatmengen gleicher Größenordnung beim Erwachsenen innerhalb von Minuten oder Stunden aus-

Tab. 2

Unter Perphyllon A nimmt die periphere Durchblutung zu (Anstieg der Haupttemperatur $\left.T_{H}\right)$. Dadurch sinkt die Blutlactatkonzentration ( $C_{\mathbf{L}}$ ). Der Lactat Pyruvat-Quotient (L/P) nähert sich den Werten für ruhende Gewebe (etwa 10).

\begin{tabular}{|c|c|c|c|c|c|c|c|c|}
\hline $\begin{array}{c}\text { Patient } \\
\text { Zeit post } \\
\text { partum }\end{array}$ & $\underset{\text { post inj: }}{\text { Min. }}$ & $\mathbf{C}_{\boldsymbol{I}}$ & $\mu \mathrm{Mol} / \mathrm{ml}$ & $\mathrm{C}_{\mathbf{P}}$ & $L / P$ & $\underset{\mathrm{mVal} / \mathrm{l}}{\mathrm{St}}\left[\mathrm{HCO}_{\mathrm{s}}\right]^{-}$ & $\begin{array}{l}\mathrm{Po}_{2} \\
\text { Torr } \\
\end{array}$ & $\mathrm{T}_{\mathbf{H}}$ \\
\hline 4 Stdn. & $\begin{array}{c}0^{*} \\
20^{* *} \\
20^{* * * *} \\
100^{* * *}\end{array}$ & $\begin{array}{l}1,960 \\
1,670 \\
1,210 \\
1,020\end{array}$ & & $\begin{array}{l}0,043 \\
0,057 \\
0,055 \\
0,050\end{array}$ & $\begin{array}{l}39,4 \\
29,4 \\
22,0 \\
21,7\end{array}$ & $\begin{array}{l}23,2 \\
23,8 \\
23,0 \\
22,0\end{array}$ & $\begin{array}{l}70,0 \\
57,2 \\
53,5 \\
63,0\end{array}$ & $\begin{array}{l}35,9^{\circ} \\
35,9^{\circ} \\
36,2^{\circ} \\
36,3^{\circ}\end{array}$ \\
\hline $\begin{array}{c}5 / 68 \\
20 \text { Min. }\end{array}$ & $\begin{array}{c}0 * \\
20 * * * \\
100 * * * \\
20 * *\end{array}$ & $\begin{array}{l}2,240 \\
0,732 \\
0,749 \\
1,090\end{array}$ & & $\begin{array}{l}0,054 \\
0,076 \\
0,041 \\
0,048\end{array}$ & $\begin{array}{r}41,2 \\
9,7 \\
18,3 \\
22,6\end{array}$ & $\begin{array}{l}18,4 \\
20,5 \\
24,5 \\
20,8\end{array}$ & $\begin{array}{l}57,0 \\
57,8 \\
44,5 \\
60,5\end{array}$ & $\begin{array}{l}35,3^{\circ} \\
35,5^{\circ} \\
35,9^{\circ} \\
35,4^{\circ}\end{array}$ \\
\hline $\begin{array}{c}2 / 68 \\
14 \text { Stdn. }\end{array}$ & $\begin{array}{c}0 * \\
20 * * * \\
100 * * *\end{array}$ & $\begin{array}{l}0,636 \\
0,409 \\
0,668\end{array}$ & & $\begin{array}{l}0,028 \\
0,022 \\
0,045\end{array}$ & $\begin{array}{l}22,9 \\
18,6 \\
14,8\end{array}$ & $\begin{array}{l}20,5 \\
22,0 \\
22,0\end{array}$ & $\begin{array}{l}56,0 \\
52,0 \\
49,5\end{array}$ & $\begin{array}{l}36,2^{\circ} \\
36,2^{\circ} \\
36,7^{\circ}\end{array}$ \\
\hline
\end{tabular}


geglichen sind $(13,14)$. Weiterhin werden - im Gegensatz zum reifen Organismus - thermo-regulatorische Belastungen des Stoffwechsels des Neugeborenen mit einem Lactatanstieg beantwortet (15). Auch erbrachte intravenöse Lactatbelastung den Beweis, daß die Lactatutilisation bei Frühgeburten mit dem Lebensalter zunimmt (16).

Schließlich findet sich Exzeßlactat auch bei reifen, durch primäre Sectio caesarea, d. h. ohne Wehentätigkeit entbundenen Kindern (17).

Diese Indizien für eine verringerte Lactatumsatzrate beim Neugeborenen werden gestützt durch Befunde an der Ratte, die eine Steigerung der Enzymaktivität des Krebszyklus in der Postnatalperiode beweisen $(18,19)$. Die klinische Verwertbarkeit der Lactat-Pyruvatbestimmung beim Neugeborenen sei an der Wirkung des Präparates Perphyllon-A, welches bei der Behandlung der Neugeborenen-Asphyxie Verwendung findet, de- monstriert: Unter Perphyllon-A $\left.(0,15 \mathrm{~m} l / \mathrm{kg} \text { i. } \mathrm{m} .)^{2}\right)$ steigt die periphere Durchblutung, wie die erhöhte Hauttemperatur des Oberschenkels $\left(\mathrm{T}_{\mathrm{H}}\right.$ ) anzeigt (vgl. (Tab. 2). In allen drei Fällen ist eine Abnahme des Blutlactates und eine Annäherung des L/P-Quotienten an normale Werte zu verzeichnen, d. h. der Reduktionszustand der Gewebe verringert sich. Das Standardbicarbonat hingegen schwankt in allen Versuchen relativ stark, so daß im Einzelfall die zu fordernde negative Korrelation des Standardbicarbonats zum Blutlactat nicht nachzuweisen ist.

Für die Übetlassung von Versuchsmengen von Perphyllon A danken wir dem Chemiewerk Homburg, Frankfurt/Main.

2) $1 \mathrm{ml}$ Perphyllon 873-A/XIII enthält:

$21,25 \mathrm{mg} 7-(\beta$ Hydroxyaethyl)-Theophyllin

$6,25 \mathrm{mg}$ Theophyllin

15,00 mg Papaverin- $\mathrm{HCl}$

$0,15 \mathrm{mg}$ Atropinmethylnitrat (Eumydrin BAYER)

\title{
Literatur
}

1. SALING, E., Das Kind im Bereich der Geburtshilfe. Georg Thieme, Stuttgart (1967). - 2. ENGström, L., P. KarLberg, G. Rooth und R. Tunell, The onset of respiration. Association for the aid of crippled children, New York, N. Y. 10017 (1966). 3. Hohorst, H. J., F. H. Kreutz und Th. Bücher, Biochem. $Z$. 332, 18 (1959). - 4. Hohorst, H. J., P. Arese, H. Bartels, D. Stratmann und H. Talke, Ann. N. Y. Acad. Sc. 119, 974 (1965). - 5. HuckaBEE, W. E., J. clin. Invest. 37, 264 (1958). 6. BRÜCK, K. und M. BRück, Pflügers Arch. Physiol. 272, 27 (1960). - 7. KintZeL, H. W., Mschr. Kinderhk. 114, 544 (1966). 8. Gercken, G., Hoppe-Seylers Z. physiol. Chem. 320, 180 (1960). - 9. Bücher, Th., R. Czok, W. Lamprecht u. E. LatzKo, in: $\mathrm{H}$. W. Bergmeyer, Grundlagen der enzymatischen Analyse, S. 253, Verlag Chemie, Weinheim, Bergstraße (1962). - 10.
BETKE, K., Der menschliche rote Blutfarbstoff, Springer-Verlag, Berlin (1954). - 11. Rootr, G., Acta paediatr. 52, 22 (1963). 12. Vedra, B., Acta paediatr. 48, 60 (1959). - 13. Margaria, R., P. Cerreteleli, P. E. di Prampero, C. Massari und G. Torelil, J. appl. Physiol. 18, 371 (1963). - 14. Hansen, J. E., G. P. Stelter und J. A. Vogel, J. appl. Physiol. 23, 523 (1967). 15. Pribylova, H. und K. ZnamenàceK, Pediatrics Springfield 37, 743 (1966). - 16. Ciampolini, M. und F. Franchini, Ann. paediatr. Basel 207, 335 (1966). - 17. ZNAMEN-ÀCEK, K. und H. Pribylova, Symp. on intrauterine dangers to the foetus, Prague, Oct 11th 14th 1966. Excerpta Medica Monograph, S. 578. 18. Potter, V. R., W. C. Schinerder und G: J. Liebl, Cancer Res. 5, 21 (1943). - 19. Dawkins, M. J. R., Proc. Roy. Soc. Med. London 150, 284 (1959).
Dr. D. Kaiser

1 Berlin 65

Reinickendorfer Str. 61

\section{Spektrum der zirkulierenden Schilddrüsenhormone und ihrer Jodderivate im menschlichen Blut}

\author{
Zur Problematik ibrer Darstellung und Identifikation
}

Von E. Zappr, G. Hoppe, M. Schmidt und F. Prange

Aus der 1. Med. Abteilung (Chefarzt: Prof. Dr. K. H. Pfeffer) des Städt. Auguste-Viktoria Krankenbauses

Berlin-Scböneberg und der Landeslebranstalt für med-techn. Assistenten Berlin (Leiter: Dr. J. Krempien)

(Eingegangen am 3. April 1968)

Herrn Prof. Dr. Dr. Ernst Schütte zum 60. Geburtstag gewidmet

Es werden die Ergebnisse einer dünnschichtchromatographischen Untersuchung der zirkulierenden Schilddrüsenhormone und ihrer Jodderivate bei einem Kollektiv normaler Probanden besprochen. Durch chemischen Nachweis gelang es, Thyroxin in $71 \%$ der Fälle darzustellen. Monojodtyrosin kommt in $62 \%$ und Dijodtyrosin in $100 \%$ der Fälle vor. Tyrosin und Thyronin, die fälschlicherweise vom Jodnachweisreagenz erfaßt werden, .kommen bei den meisten Probanden vor. Durch ihr chromatographisches Verhalten lassen sich gelegentlich auftretende unbekannte Flecken in zwei Gruppen einordnęn. Auf die mögliche Bedeutung der zirkulierenden Jodtyrosine wird hingewiesen. Die Rolle der nicht jodierten Tyrosine und Thyronine als Fehlerquelle bei der dünnschichtchromatographischen Identifikation ihrer halogenierten Derivate wird erklärt.

The circulating thyroid hormone and related iodo-compounds were studied by thin layer chromatography in a group of normal probands. Using chemical detection methods, thyroxin was found in $71 \%$, monoiodotyrosine in $62 \%$, and diiodotyrosine in $100 \%$ of the probands. Tyrosine and thyronine, which also react with the iodine-locating reagent, were present in most of the probands. Some unknown spots, which occasionally occur, can be classified into two groups according to their chromatographic behaviour. The possible significance of the circulating iodotyrosine is discussed. The error caused by the reaction of non-iodinated tyrosine and thyronine in the thin layer chromatographic identification of the halogenated derivatives is explained. 